\title{
Insight into the effects of confined hydrocarbon species on the lifetime of methanol conversion catalysts
}

I. Lezcano-Gonzalez ${ }^{1,2^{*}}$, E. Campbel1 ${ }^{1,2}$, A. E. J. Hoffman ${ }^{3}$, M. Bocus ${ }^{3}$, I. V. Sazanovich ${ }^{4}$, M. Towrie $^{4}$, M. Agote-Aran ${ }^{1,2}$, E. K. Gibson ${ }^{2,5}$, A. Greenaway ${ }^{1,2}$, K. De Wispelaere ${ }^{3}$, V. Van Speybroeck $^{3 *}$, A. M. Beale l, $^{1,2}$

${ }^{1}$ Chemistry Department, University College London, London, WC1H 0AJ, UK.

${ }^{2}$ UK Catalysis Hub, Research Complex at Harwell, Didcot, OX11 0FA, UK.

${ }^{3}$ Center for Molecular Modeling, Ghent University, Technologiepark 46, 9052 Zwijnaarde, Belgium

${ }^{4}$ Central Laser Facility, STFC, Research Complex at Harwell, Didcot, OX11 0QX, UK.

${ }^{5}$ School of Chemistry, University of Glasgow, Glasgow, UK

*Correspondence to: i.lezcano-gonzalez@ucl.ac.uk; veronique.vanspeybroeck@ugent.be; Andrew.beale@ucl.ac.uk

\begin{abstract}
The methanol-to-hydrocarbons (MTH) reaction refers collectively to a series of important industrial catalytic processes to produce either olefins (MTO) or gasoline (MTG). Mechanistically, methanol conversion proceeds via a 'pool' of hydrocarbon species. For MTO, these can broadly be delineated into 'desired' lighter olefins and 'undesired' heavier fractions causing deactivation in a matter of hours. The crux in further catalyst optimization is the ability to follow the formation of carbonaceous species during operation. Herein, we report the combined results of an operando Kerr-Gated Raman Spectroscopy study with stateof-the-art operando molecular simulations, which allowed to follow the formation of hydrocarbon species at various stages of methanol conversion. Polyenes are identified as crucial intermediates towards formation of polycyclic aromatic hydrocarbons, with their fate being largely determined by the zeolite topology. Notably, we provide the missing link between active and deactivating species, which allows us to propose potential design rules for future generation catalysts.
\end{abstract}

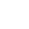


'building blocks' for the global polymer and plastic market, giving a propylene market worth of $\$ 90 \mathrm{~b} \mathrm{p.a.}{ }^{1}$ Steam cracking of light hydrocarbons or naphtha typically produces ethylene, whereas fluid catalytic cracking of naphtha produces propylene. Alternative, sustainable routes to olefin production are necessary to omit crude oil as a source, requiring new process technologies and catalytic materials. Alternative sources for olefins production include coal and natural gas, and sustainable options, i.e. second-generation biomass, which can be realized at a large scale through a multi-step process involving methanol synthesis from syngas, followed by the methanol-to-hydrocarbon (MTH) process using zeolites. ${ }^{2-4}$ MTH has been commercialized in many variants and remains a vital technology for the production of chemicals and fuels, especially under a variable window of operation in terms of feed or product distribution. The scope to influence MTH products by altering parameters such as reaction conditions (temperature and flow) and zeolite properties (topology and acidity) is particularly attractive. These variables sway product selectivity between the extremities of gasoline (methanol-to-gasoline or MTG) and light olefins (MTO). ${ }^{2,3}$ Catalyst stability is perhaps the major challenge for the widespread use of this technology, specifically the fast formation of carbon deposits during operation severely compromises process efficacy. ${ }^{2,5-7}$ For small-pore zeolites, deactivation occurs in a matter of hours. Despite intensive research efforts, a comprehensive molecular insight into deactivation phenomena is still absent yet necessary to improve process efficiency. The presence of many intermediate species and need to track them under reaction conditions renders this very challenging. ${ }^{2,3,5}$

To set the scene, the MTH reaction has been shown to proceed through an indirect hydrocarbon pool (HP) mechanism, wherein organic molecules confined within zeolite micropores act as reaction centres to which methanol is added and olefins are split off via dealkylation/cracking steps (Fig. 1a). ${ }^{2,8,9}$ There is a consensus that methylated benzenes and olefins are key HP species in both CHA and MFI zeolite topologies, whereas polycyclic aromatic hydrocarbons (PAH) are related to catalyst deactivation, blocking zeolite pores and forming carbon deposits. ${ }^{2,5,8-12}$ Recent studies have shown that altering the catalyst structure and/or acidity, as well as co-feeding of $\mathrm{H}_{2}$ can improve lifetime, ${ }^{4,13,14}$ nevertheless, it is unclear how the plethora of adsorbed species affects this. Most mechanistic insight has been gleaned from NMR, ${ }^{15-18}$ Raman, ${ }^{19-21}$ IR, $^{10,22,23}$ and UV-vis spectroscopic studies, ${ }^{23-25}$ some of which were employed under in situ/operando conditions, as well as theoretical calculations. ${ }^{11,26}$ Studies that can truly discriminate between essential and deactivating species in the catalytic process are very limited; ${ }^{15,20,21,23-25,27}$ with time/temperature-resolved studies 
most suited to unravel this complexity. To further progress in controlling catalytic performance, a deep mechanistic understanding into the formation and evolution of the HP species under operating conditions is needed.



Figure 1. Illustration of the different stages of the MTO reaction and the Kerr-Gate Raman setup. a. Illustration of different stages of the MTO reaction on CHA-type zeolite materials, with the corresponding adsorbed intermediates and outlet products. b. Representation of the Kerr-Gate blocking fluorescence from the detector. This technique makes use of the difference between fluorescence and Raman temporal behavior (i.e. Raman process is instant and limited by the laser pulse while fluorescence occurs on longer time scale of $10^{-6}$ to $10^{-9} \mathrm{~s}$ ) to temporally reject fluorescence (see Supporting Information for further details).

Herein we combine operando Kerr-Gated Raman studies with advanced simulation tools to follow carbonaceous species formation during methanol conversion. Raman spectroscopy is a powerful technique for characterizing carbon species under reaction conditions, but its application to zeolite catalysts is often hindered by inherent and incidental fluorescence from the sample. ${ }^{28}$ To some extent using differing excitation wavelengths can circumvent this, though at the expense of either weaker Raman signal (IR source) or higher energy load on the 
sample (UV) which can cause photodegradation. ${ }^{28,29}$ An interesting alternative is to use a visible excitation source combined with a Kerr-Gated spectrometer, which discriminates between the Raman signal and fluorescence based on the temporal differences of the two processes (Fig. 1b and S1) ${ }^{30,31}$ Furthermore since many key molecular 'protagonists' in the reaction pathway are colored ${ }^{23,24}$ the phenomenon of resonance enhancement of Raman (up to $10^{8}$ signal enhancement) allows us to detect species not normally observable in this reaction. $^{28,29}$ The benefit of performing Kerr-Gated Raman spectroscopy vs. conventional Raman is clearly demonstrated in Fig. S2. Operando molecular simulations identified reaction intermediates and determined their mobility within the zeolite host. The combined experimental/theoretical approach allowed the hydrocarbon evolution to be pieced together within two of the most well-studied zeolite topologies: CHA and MFI. As a major finding, we identified the crucial role of polyenes and show that their fate is dependent on the zeolite topology. Within the CHA topology, they are precursors for deactivating PAH species.

\section{Early stages of the MTO reaction: pre-equilibrium stage and initiation of the induction} period $\left(100\right.$ to $\left.260{ }^{\circ} \mathrm{C}\right)$. The evolution of hydrocarbon species formed during MTO on SSZ13 was examined by operando Kerr-Gated Raman Spectroscopy combined with real-time product analysis using a mass spectrometer (MS) (see Methods and Table S1 for further details). Data recorded up to $300{ }^{\circ} \mathrm{C}$ are given in Fig. 2 and S3-4. Between 100 and $240{ }^{\circ} \mathrm{C}$, a gradual drop in the Raman bands of methanol (2954 and $2855\left(\mathrm{C}-\mathrm{H} v_{\mathrm{as}}\right.$ and $\left.\mathrm{v}_{\mathrm{s}}\right), 1455\left(\mathrm{CH}_{3} \delta\right)$, and $1006 \mathrm{~cm}^{-1}$ (C-O v)) was observed (Fig. S3-4), with dimethyl ether (DME) and water detected by MS in the reactor outlet stream (Fig. S4). These products are characteristic of the pre-equilibrium stage of the reaction (Fig. 1) ${ }^{32}$ wherein methanol is dehydrated into a mixture of methanol, DME and water. Note that methanol bands in the experimental spectra are in agreement with the simulations (see Supporting Information, Fig. S5-S6, Table S2 and Fig. S7-S8). At $240-260{ }^{\circ} \mathrm{C}$ the bands of methanol were barely observed (Fig. 2a), and a new weak signal was detected at $1600-1625 \mathrm{~cm}^{-1}$ that according to previous work ${ }^{33,34}$ and our simulations (Fig. 3a and S9-11), can be assigned to stretching modes of isolated $\mathrm{C}=\mathrm{C}$ bonds, indicating the onset of alkene formation and possibly, small polyenes. These observations are consistent with the formation of the first hydrocarbons, ${ }^{16}$ representing the beginning of the induction period and the build-up of HP species within zeolite micropores (Fig. 1). 

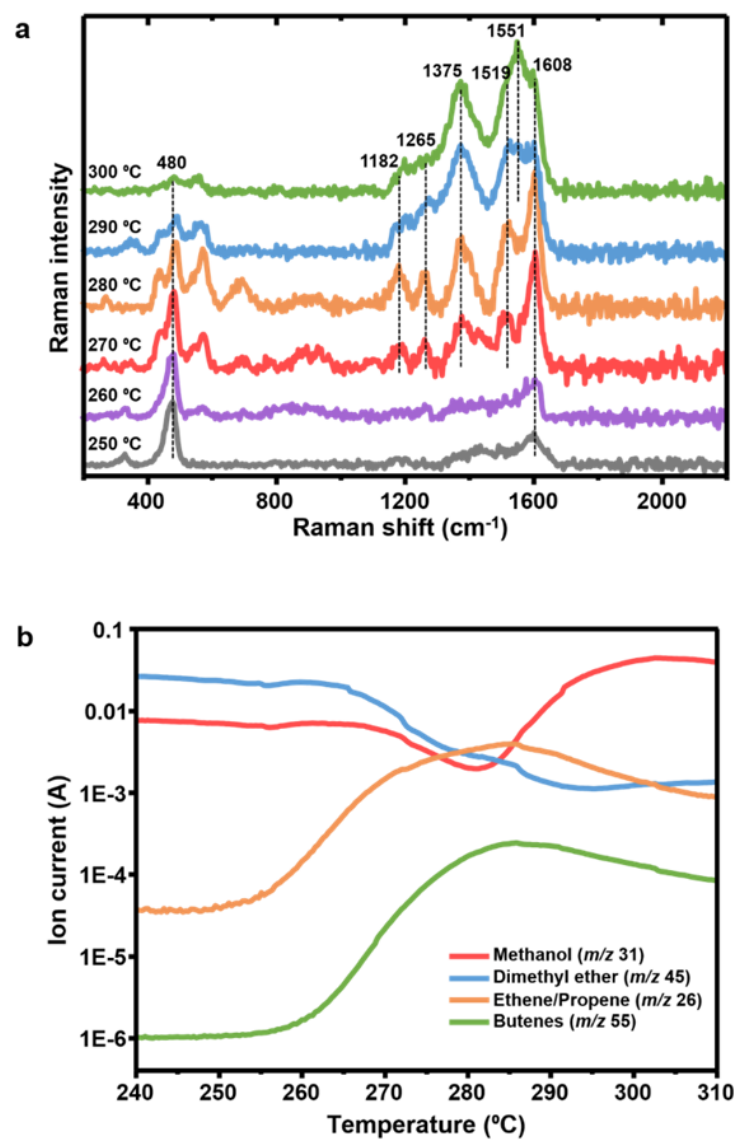

Figure. 2. Operando Kerr-Gated Raman of SSZ-13 zeolite during the MTO reaction Formation of HP intermediates and onset of deactivation. a. Kerr-Gated Raman spectra of SSZ-13 zeolite during the MTO reaction between 250 and $300{ }^{\circ} \mathrm{C}$. Measurements performed at increasing reaction temperatures (heating rate $1^{\circ} \mathrm{C} \cdot \mathrm{min}^{-1}$; WHSV of $1.6 \mathrm{~g} \mathrm{geOH}_{\mathrm{H}} \cdot \mathrm{g}_{\mathrm{cat}}{ }^{-1} \cdot \mathrm{h}^{-1}$ ). Excitation wavelength $400 \mathrm{~nm}$. Raman bands are observed at $1608\left(\mathrm{C}=\mathrm{C} \quad v_{\mathrm{s}}\right), 1551$ (conjugated $\pi$-system $\mathrm{C}=\mathrm{C} v), 1519(\mathrm{C}=\mathrm{C} \mathrm{v}$ as $), 1375\left(\mathrm{CH}_{2} / \mathrm{CH}_{3} \delta\right.$; ring breathing mode), 1265 (C-H rock), 1182 (C-C v), 576 (C-C $\delta$ ) and $480 \mathrm{~cm}^{-1}$ (zeolite T-O bend vibration). b. Mass traces of methanol and the reaction products.

Identification of $\mathrm{HP}$ intermediates $\left(270\right.$ to $\left.280{ }^{\circ} \mathrm{C}\right)$. Further temperature increase led to significantly increased methanol conversion - with a maximum at $283{ }^{\circ} \mathrm{C}$, along with the formation of $\mathrm{C}_{2}-\mathrm{C}_{4}$ products (Fig. 2b), characteristic of the autocatalytic stage (Fig. 1). In accordance, intense Raman bands observed in the spectra at $270-280{ }^{\circ} \mathrm{C}$, attributed to various HP species. Previous work in conjunction with our simulations (Fig. 3 and S12), 19,33,34 identified bands at ca. 1608 and $1519\left(\mathrm{C}=\mathrm{C} v_{\mathrm{s}}\right.$ and $\left.v_{\text {as }}\right), 1375\left(\mathrm{CH}_{3} \delta\right), 1265(\mathrm{C}-\mathrm{H}$ rock $), 1182$ (aromatic C-C $v$ modes), $576 \mathrm{~cm}^{-1}$ (alkyl aromatic ring $\mathrm{C}-\mathrm{C}_{\delta}$ ); the origin of these bands we 
132 attribute to methylated-benzenium ions which are now readily observable due to resonance 133 enhancement. ${ }^{17,24} \mathrm{We}$ note the excellent correspondence between our experiments and 134 theoretical simulations (Fig. 3b and S12).

135 Besides alkenes, small polyenes and methylated-benzenium ions, our simulations identified 136 contributions from additional species. Specifically, Raman bands around $550 \mathrm{~cm}^{-1}\left(\mathrm{C}-\mathrm{C}_{\delta}\right)$ and 137 between $1250-1400 \mathrm{~cm}^{-1}\left(\mathrm{C}-\mathrm{H}\right.$ rock and $\mathrm{CH}_{2}$ and $\left.\mathrm{CH}_{3} \delta\right)$ characteristic of dienylic cations 138 (Fig. 3 and S9), formed from dienes by protonation and subsequent hydride abstraction or the 139 reaction of monoenyl carbocations and olefins. The identification of (methylated140 )naphthalene(s) is more challenging. We note that the width and height of the $1375 \mathrm{~cm}^{-1}$ band 141 is sufficiently broad to contain multiple contributions including, as predicted by theory (Fig. 3 142 and S12) and identified in previous works, ${ }^{33}$ a ring-breathing mode of naphthalenes along 143 with $\mathrm{CH}_{3} \delta$ modes. From these observations, methylated-benzenium ions, along with alkenes, 144 small polyenes, dienylic cations and possibly (methylated-)naphthalene(s) are concluded to be 145 the HP species formed on SSZ-13 during the induction period and present in the autocatalytic 146 stage of the reaction. 

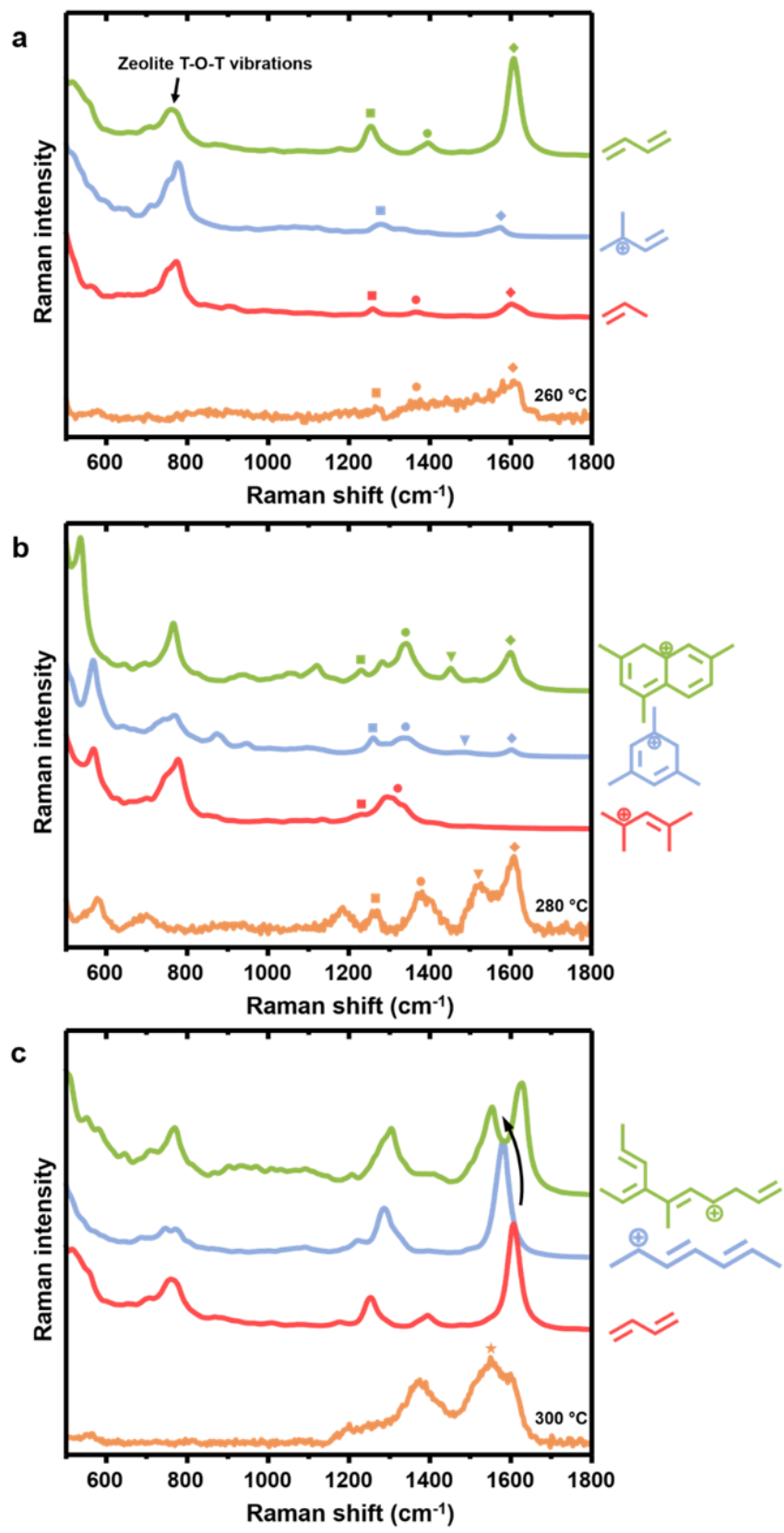

Figure. 3. Comparison of experimental and simulated Raman spectra. a. Simulated Raman spectra of SSZ-13 zeolite loaded with model alkenes and small polyenes (red, blue and green traces) vs. experimental Raman spectrum (orange trace) acquired during the MTO reaction at $260^{\circ} \mathrm{C}$. b. Simulated Raman spectra of SSZ-13 zeolite loaded with model dienyl, benzene and naphthalene species (red, blue and green traces) vs. experimental Raman spectrum (orange trace) acquired during the MTO reaction at $280{ }^{\circ} \mathrm{C}$. c. Simulated Raman spectra of SSZ-13 zeolite loaded with model polyenes (red, blue and green traces) vs. experimental Raman spectrum (orange trace) acquired during the MTO reaction at 300 ${ }^{\circ} \mathrm{C}$. Protonated structures are indicated with a + sign. Full assignments are provided in the ESI, along with the simulated spectra of additional model alkenes, polyenes and aromatic 
species.

Confined hydrocarbon species at the onset of deactivation $\left(290\right.$ to $\left.300{ }^{\circ} \mathrm{C}\right)$. Coincident with decreasing methanol conversion at $290{ }^{\circ} \mathrm{C}$, a new intense band at $1551 \mathrm{~cm}^{-1}$ emerged in the $\mathrm{C}=\mathrm{C}$ stretch region of conjugated olefins (Fig. 2). Bands in this region were previously observed during conversion of methanol, DME and light olefins, and tentatively assigned to polyene-type species. ${ }^{19,22,33}$ However, their high reactivity has prevented the study of zeolite adsorbed polyenes, preventing their unambiguous identification. To gain definitive insight into the species present, vibrational signatures of a number of polyene species were calculated (Fig. 3c and Figs. S9-11, S13-19). For linear polyenes with either straight or curled topologies, four characteristic vibrations were identified, including the stretching mode of the conjugated $\pi$-system $\left(1520-1580 \mathrm{~cm}^{-1}\right)$, which red-shifted with increasing chain length. Branched polyenes showed an additional feature at $850-1000 \mathrm{~cm}^{-1}$, due to the out-of-plane wagging of $\mathrm{C}-\mathrm{H}$ bonds. The absence of this mode in the experimental spectrum, along with the stretching frequency of the conjugated $\pi$-system identifies extended polyenes with no or small branches (Fig. 3c and Figs. S9-11),

As the reaction progresses, at $300{ }^{\circ} \mathrm{C}$, significant growth of the $1551 \mathrm{~cm}^{-1}$ band was observed. No additional features were detected in the $850-1000 \mathrm{~cm}^{-1}$ region (Fig. 3). The intensification of the $1551 \mathrm{~cm}^{-1}$ band accompanied an important decrease in methanol conversion (Fig. 2b), revealing a direct correlation between extended polyenes formation and the onset of catalyst deactivation, likely associated with confinement-induced effects on the mobility of these large molecules.

Mobility of hydrocarbon species formed during onset of activation and deactivation. So far, it remains unclear whether the carbonaceous species detected block the zeolite pore system or remain mobile. First principle molecular dynamics simulations (MD) allowed the examination of the mobility of detected hydrocarbons within the zeolite pores (Fig. S20-33). We followed the path of the centres of mass (COMs) of the carbon atoms during MD runs of 40 ps at $350^{\circ} \mathrm{C}$, projecting the $\mathrm{COMs}$ onto the different channels as the reaction intermediates are expected to move along channels in the zeolite. The resulting mobility plots are visualized through a dark orange line in a 2D-plot (Fig. 4 and Fig. S21, S25, S28, S30 and S31) and the projections of all carbon atom positions on the channel are visualized in light orange. Marked differences in mobility for the various HP species were observed. With small alkenes, simulations indicated no obvious impediments to their mobility, although a lack of preferred 

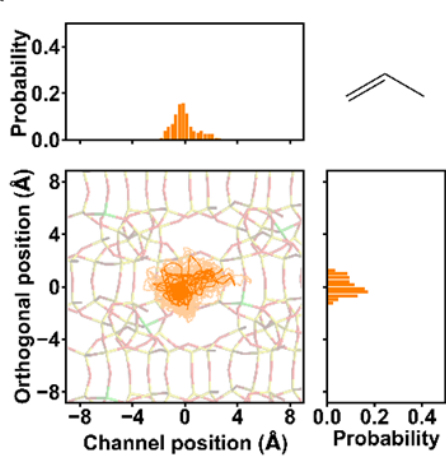
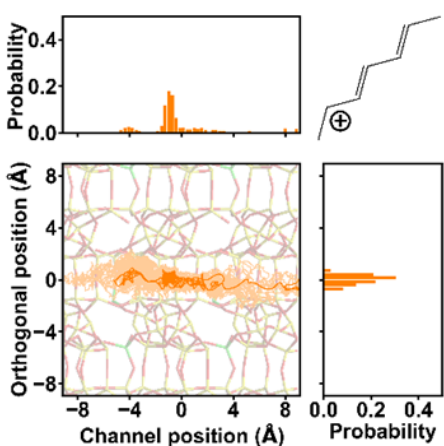

\section{政}
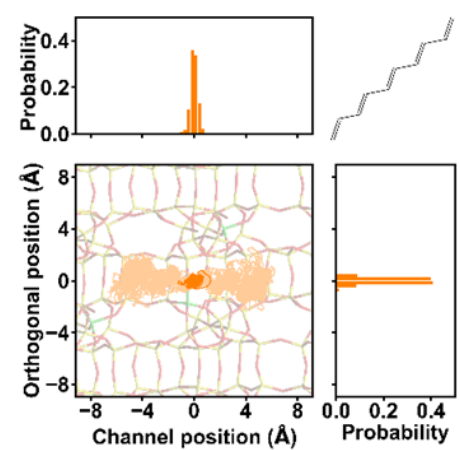

Figure 4. Mobility of polyene species formed at the onset of deactivation. Position of the centre of mass of the intermediate (dark orange) and the positions of the carbon atoms (light orange) projected on one of the 8-ring channels (horizontal axis) and a direction perpendicular to it (vertical axis). The amount of time the centre of mass resides at a certain position is illustrated by histogram plots. a. Propene. b. Heptatriene. c. Decapentaene. Protonated structures are indicated with a + sign.

Formation of polycyclic aromatic hydrocarbons $\left(310\right.$ to $\left.450{ }^{\circ} \mathrm{C}\right)$. Further increase in temperature (Fig. 5a) saw the evolution of clear, distinctive low-frequency bands (629 to 1100 $\mathrm{cm}^{-1}$ ) and more subtle changes in the intensities of bands between 1250 and $1608 \mathrm{~cm}^{-1}$. Based on our simulations, these features are consistent with the formation of PAH (Fig. 5b and S12). 
The decreased relative intensity of the $1608 \mathrm{~cm}^{-1}\left(v_{\mathrm{s}} \mathrm{C}=\mathrm{C}\right)$ shoulder is likely due to consumption of small alkenes/polyenes and/or methylated-benzenium ions. While PAH also present a band around this frequency (Fig. 5b), these are less resonant than the species being consumed leading to a decrease in signal intensity. Additionally, a drop in the zeolite bands, essentially vanishing by $360{ }^{\circ} \mathrm{C}$, indicates significant hydrocarbon accumulation, due to increased absorption (gradual sample darkening) of the incident excitation beam. ${ }^{36}$

Our mobility studies revealed that branched polyenes block the CHA pore system. We observed during a regular $\mathrm{MD}$ run at $350{ }^{\circ} \mathrm{C}$ of an exemplar branched polyene $(3,4-$ divinylhexa-1,3,5-triene) spontaneous intramolecular cyclisation, forming a new ring type species (in this case 3,4-diethyl-2-methylcyclopentenium ion). This observation establishes for the first time a clear link between the presence of polyenes and the eventual formation of PAHs (Fig. S13). To assess whether this propensity toward cyclisation is intrinsic to all polyenes irrespective of their degree of branching, we performed additional MD simulations on the non-branched, 6-protonated 1,3,5-heptatriene at $350^{\circ} \mathrm{C}$. When starting from a cis configuration for the $\mathrm{C}_{1}-\mathrm{C}_{2}$ and $\mathrm{C}_{2}-\mathrm{C}_{3}$ bonds, we observed spontaneous cyclisation in all simulations (Fig. S34a), confirming that intramolecular cyclisation from the cis, cis conformation is basically barrierless. Steric hindrance between the $\mathrm{C}_{1}$ and the $\mathrm{C}_{5}$ hydrogens explains the high reactivity of the $c i s$, cis conformation, preventing the $\pi$-system being fully coplanar and significantly reducing the delocalization of the positive charge (Fig. S34b). However, linear, non-branched polyenes must overcome an energetic penalty to isomerize the $\mathrm{C}_{1}-\mathrm{C}_{2}$ and $\mathrm{C}_{2}-\mathrm{C}_{3}$ bonds from the stable trans, trans to the reactive cis, cis conformation. For non-branched polyenes, this barrier equals $55 \mathrm{~kJ} / \mathrm{mol}$, whereas highly branched polyenes have no energetic barrier to form the pre-reactive complex for intramolecular cyclisation (Figs. S35-36). We conclude that branched polyenes formed in the zeolite pores immediately cyclize, whereas linear polyenes survive longer due to the energetic penalty to form the reactive cis, cis isomers.

Besides intramolecular cyclisation, other ring-forming routes from extended polyenes comprise: electrophilic aromatic substitution of single-ring aromatics or Diels-Alder reaction between polyenes and alkenes, both followed by intramolecular rearrangement steps (Figure S37). In view of the concomitant consumption of small alkenes/polyenes and methylatedbenzenium ions during this temperature range (cause of the decreased band intensity at 1608 $\mathrm{cm}^{-1}$ ), it is likely these other processes also occur. Once extended PAHs form, they block the 
CHA cage, as supported by a mobility analysis (Fig. S28-29). Operando Kerr-Gated Raman is able therefore to identify initial polyene formation, track their consumption and consequent transformation into PAH. This is seen both under variable-temperature (Fig. 2) and isothermal conditions (Fig. S38). We propose therefore that deactivation of CHA materials occurs via cage blocking first by extended polyenes and subsequently as these react, PAH species.
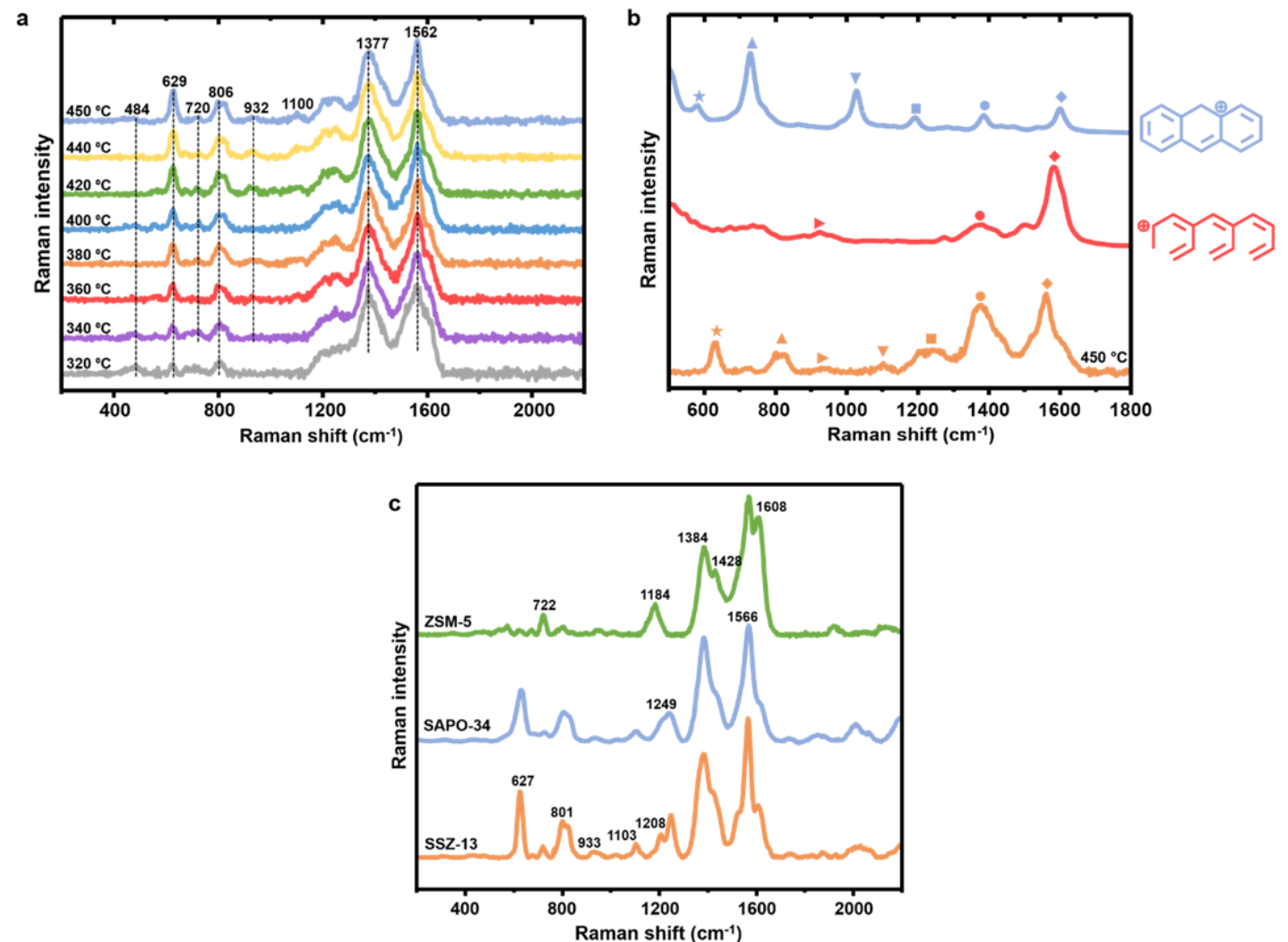

Figure. 5. Operando Kerr-Gated Raman of SSZ-13 zeolite during the MTO reactionFormation of polycylic aromatic hydrocarbons and comparison with ZSM-5 and SAPO34- archetypal MTH catalysts. a. Kerr-Gated Raman spectra of SSZ-13 zeolite during the MTO reaction between 320 and 450 C. Measurements performed at increasing reaction temperatures (heating rate $1{ }^{\circ} \mathrm{C} \cdot \mathrm{min}^{-1}$; WHSV of $1.6 \mathrm{~g}_{\mathrm{MeOH}} \cdot \mathrm{g}_{\mathrm{cat}}{ }^{-1} \cdot \mathrm{h}^{-1}$ ). Excitation wavelength $400 \mathrm{~nm}$. Raman bands are observed at $1608(\mathrm{C}=\mathrm{C} \mathrm{v}$ ), 1562 (conjugated $\pi$-system $\mathrm{C}=\mathrm{C} v$ ), $1377\left(\mathrm{CH}_{2} / \mathrm{CH}_{3} \delta\right.$; ring breathing mode), 1265 (C-H rock), 1100-629 (ring-opening vibrations) and $480 \mathrm{~cm}^{-1}$ (zeolite T-O bend vibration). b. Simulated Raman spectra of SSZ-13 zeolite loaded with a model polycyclic aromatic (anthracene, blue trace) and its precursor (red trace) vs. experimental Raman spectrum acquired during the MTO reaction at $450{ }^{\circ} \mathrm{C}$ (orange trace). Protonated structures are indicated with a + sign. Full assignments are provided in the ESI, along with the simulated spectra of additional model alkenes, polyenes and aromatic 
species. c. Kerr-Gated Raman spectra of SSZ-13, SAPO-34 and ZSM-5 catalysts recorded at room temperature after quenching the reaction at $450 \mathrm{C}$.

Effect of zeolite topology on intermediates formation. Similar results were obtained during MTH over ZSM-5, although formation of HP species occurs at higher temperatures (i.e. 300 ${ }^{\circ} \mathrm{C}$; Fig. S39a). Above $300{ }^{\circ} \mathrm{C}$, features at $1605,1522,1383,1265,1179$ and $564 \mathrm{~cm}^{-1}$ were observed, assigned to methylated-benzenium ions, dienyl carbocations and possibly (methylated-)naphthalene(s). Simultaneously, the MS showed a gradual increase in methanol conversion, olefins and aromatics formation (Fig. S39b). At $330^{\circ} \mathrm{C}$, polyenes are formed (i.e. $1552 \mathrm{~cm}^{-1}$ ) without any effect on activity. Also contrasting to SSZ-13, the lower relative intensity of this band to $1605 \mathrm{~cm}^{-1}\left(\mathrm{C}=\mathrm{C} \mathrm{v}_{\mathrm{s}}\right)$ suggests that among the species detected, methylated-benzenium ions dominate. Importantly, further heating to $450{ }^{\circ} \mathrm{C}$ (Fig. S39c) did not induce PAH formation - consistent with previous reports that PAHs do not build-up significantly within ZSM-5 micropores at this stage, ${ }^{37}$ and no drop in methanol conversion/product formation was observed by MS. We propose that in larger-channelled ZSM-5, linear polyenes diffuse relatively unimpeded, preventing catalyst deactivation through blocking. Considering the larger void volume of the chabazite cage compared with the ZSM-5 channel intersections, ${ }^{38}$ steric constraints may also play a role, inhibiting polyene cyclization on the MFI topology and preventing PAH formation. The zeolite band at $360 \mathrm{~cm}^{-1}$ also did not drop, indicating a diminished accumulation of hydrocarbons compared to SSZ13. Note however, that due to the increased lifetime of this material, ${ }^{37}$ we cannot rule out that at longer reaction times, polyenes might contribute to deactivation.

Spent catalysts. At $450{ }^{\circ} \mathrm{C}$, the reactions were quenched to record several scans at room temperature (Fig. 5c). Again, no clear features of PAH were seen for ZSM-5 - supporting the idea that, if present, just little amounts of PAH are formed on this catalyst, probably on the external zeolite surface. ${ }^{39}$, while SSZ-13 showed a number of intense low-frequency peaks, attributable to PAH. For comparison, data are also given for (CHA) SAPO-34, considered the archetypal system for industrial MTO. ${ }^{2}$ The final spectra for SAPO-34 and SSZ-13 are almost identical, confirming that the mechanistic insight is applicable to CHA topologies in general. The only noticeable difference between these catalysts is the temperature of HP species formation $\left(270{ }^{\circ} \mathrm{C}\right.$ for SSZ-13 vs. $300{ }^{\circ} \mathrm{C}$ for SAPO-34), arising from the weaker acidity of 
the silicoaluminophosphate, as previously reported. ${ }^{24,40}$ For completeness, the Kerr-Gated Raman data for MTO on SAPO-34 are given in Fig. S40, with additional characterization on the spent SSZ-13 (Figs. S41-42).

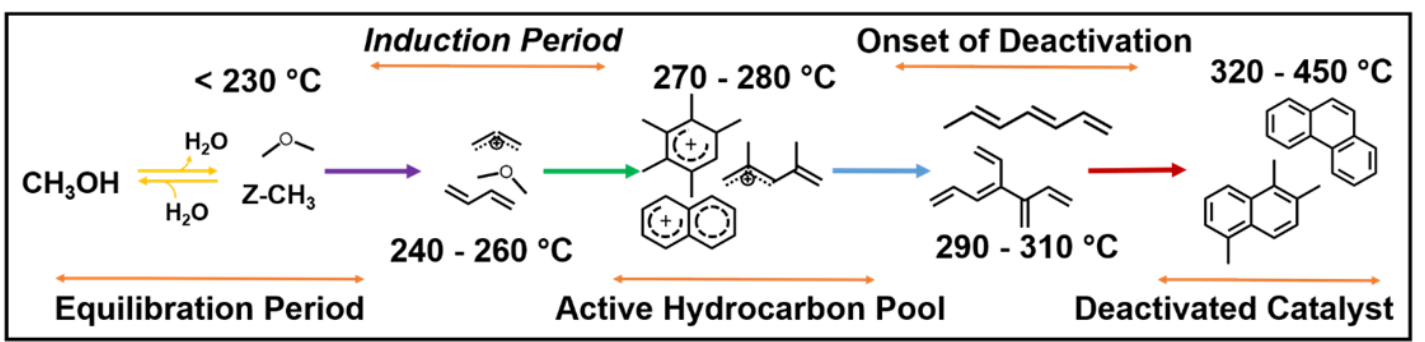

Figure. 6. Hydrocarbon species evolution taking place during the MTO reaction on small-pore catalyst materials. Different hydrocarbon pool species are formed during the induction period of the MTO reaction, including alkenes, small polyenes, dienylic cations, methylated-benzenium ions and possibly, (methylated-)naphthalene(s), which are responsible for the formation of $\mathrm{C}_{2}-\mathrm{C}_{4}$ alkenes in the period of maximum methanol conversion. The formation of polyenes with no or small branches (e.g. decapentaene) - which show almost no mobility and curl around the 8-ring of the zeolite framework, leads to an important decrease in activity, likely related to a reduced diffusivity of both reactant and products. Subsequently, $\mathrm{PAH}$ start to accumulate on the catalyst surface, formed from polyenes (possibly) via intramolecular cyclisation.

Outlook. The unique combination of Kerr-Gated Raman Spectroscopy with molecular simulations allowed the formation of hydrocarbon species at various stages of methanol conversion to be followed (Fig. 6). Our results conclude the CHA topology rapidly deactivates due to the tendency of extended polyenes to block the zeolite pores, eventually undergoing cyclisation within the large chabazite cage and leading to the formation of PAH. We note that polyenes have been previously proposed to provide the precursors to PAH that lead to deactivation. ${ }^{33,41}$ However, for the first time we provide mechanistic insight into how and why this occurs and propose that this does not happen in the MFI structure due to steric constraints inhibiting the cyclisation of polyenes and PAHs within the pores and channel intersections leading to enhanced catalyst lifetime (Fig. 6). Previous studies demonstrated that SAPO-34 deactivation may start before significant amounts of PAH form, possibly arising from bulky products trapped in the large framework cavities. ${ }^{6}$ Our findings evidence that it is the extended polyenes with no or small branches that are the crucial deactivating species causing the onset of deactivation. As polyenes are typically produced through H-transfer reactions, higher reaction temperatures and/or diminished acid site densities should be 
beneficial, by favouring reactions such as cracking or reducing the rate of $\mathrm{H}$-transfer. Nonetheless, to ultimately design a stable catalyst, steric effects also need to be fully considered.

Based on our findings, optimal MTO catalysts must present topological characteristics similar to chabazite (i.e. 8-ring three-dimensional channels to retain shape-selectivity, maximising light olefins production while enabling diffusion), ideally with smaller cavities approaching the size of MFI channel intersections, to inhibit intramolecular polyene cyclisation. Materials which combine small cavities to prevent cyclisation reactions and tailored acid sites to decrease $\mathrm{H}$-transfer rate will be less prone to deactivation via extended polyene formation and more competitive for MTO. An examination of the zeolite database suggests that there is perhaps only one 8-ring three dimensional zeolite (MER) with the cage size/aspect ratio required to inhibit polyene cyclisation. A combination of this topology only being available with a low $\mathrm{Si}: \mathrm{Al}$ and the comparatively narrow pore openings will likely affect the olefin product distribution however. ${ }^{38}$ Nowadays, hypothetical databases exist that contain a huge numbers of structures for exploration; an examination of one database reveals already a few a interesting 3-dimensional, 8-ring containing candidate structures that potentially combine restricted cage size with accessibility similar to CHA (3.8 $\AA$ ) (Fig. S43). ${ }^{42,43}$ The challenge though is to make such structures, although we note that our results indicate how insight derived from reaction mechanisms can provide a basis for targeted zeolite design (an approach recently advocated by Corma et $a l .{ }^{13}$ ) which, when combined with potential hypothetical structures, could allow for the realization of a solution to this 'polyene problem'. Understanding a reaction mechanism lies at the heart of improving a catalyst's performance suggesting that this approach could prove very powerful for revealing the 'mechanistic secrets' behind a range of catalytic reactions. The technique is not limited to catalysis however and could easily be applied to study a range of functional materials.

\section{References}

1. Available at: https://www.transparencymarketresearch.com/ethylene-and-propylene.html.

2. Olsbye, U. et al. Conversion of Methanol to Hydrocarbons: How Zeolite Cavity and Pore Size Controls Product Selectivity. Angew. Chemie Int. Ed. 51, 5810-5831 (2012).

3. Yarulina, I., Chowdhury, A. D., Meirer, F., Weckhuysen, B. M. \& Gascon, J. Recent trends and fundamental insights in the methanol-to-hydrocarbons process. Nat. Catal. 1, 398-411 (2018). 
4. Yarulina, I. et al. Structure-performance descriptors and the role of Lewis acidity in the methanol-to-propylene process. Nat. Chem. 10, 804-812 (2018).

5. Olsbye, U. et al. The formation and degradation of active species during methanol conversion over protonated zeotype catalysts. Chem. Soc. Rev. 44, 7155-7176 (2015).

6. Hereijgers, B. P. C. et al. Product shape selectivity dominates the Methanol-to-Olefins (MTO) reaction over H-SAPO-34 catalysts. J. Catal. 264, 77-87 (2009).

7. Bleken, F. et al. The Effect of Acid Strength on the Conversion of Methanol to Olefins Over Acidic Microporous Catalysts with the CHA Topology. Top. Catal. 52, 218-228 (2009).

8. Dahl, I. M. \& Kolboe, S. On the Reaction Mechanism for Hydrocarbon Formation from Methanol over SAPO-34: I. Isotopic Labeling Studies of the Co-Reaction of Ethene and Methanol. J. Catal. 149, 458-464 (1994).

9. Dahl, I. M. \& Kolboe, S. On the Reaction Mechanism for Hydrocarbon Formation from Methanol over SAPO-34: 2. Isotopic Labeling Studies of the Co-reaction of Propene and Methanol. J. Catal. 161, 304-309 (1996).

10. Bjørgen, M. et al. Spectroscopic Evidence for a Persistent Benzenium Cation in Zeolite HBeta. J. Am. Chem. Soc. 125, 15863-15868 (2003).

11. McCann, D. M. et al. A Complete Catalytic Cycle for Supramolecular Methanol to Olefins Conversion by Linking Theory with Experiment. Angew. Chemie Int. Ed. 47, 5179-5182 (2008).

12. Fu, H., Song, W. \& Haw, J. F. Polycyclic Aromatics Formation in HSAPO-34 During Methanol-to-Olefin Catalysis: Ex Situ Characterization After Cryogenic Grinding. Catal. Letters 76, 89-94 (2001).

13. Li, C. et al. Synthesis of reaction adapted zeolites as methanol-to-olefins catalysts with mimics of reaction intermediates as organic structure directing agents. Nat. Catal. 1, 547-554 (2018).

14. Arora, S. S., Nieskens, D. L. S., Malek, A. \& Bhan, A. Lifetime improvement in methanol-toolefins catalysis over chabazite materials by high-pressure H2 co-feeds. Nat. Catal. 1, 666-672 (2018).

15. Hunger, M., Seiler, M. \& Buchholz, A. In situ MAS NMR spectroscopic investigation of the conversion of methanol to olefins on silicoaluminophosphates SAPO-34 and SAPO-18 under continuous flow conditions. Catal. Letters 74, 61-68 (2001).

16. Wang, W., Buchholz, A., Seiler, M. \& Hunger, M. Evidence for an Initiation of the Methanolto-Olefin Process by Reactive Surface Methoxy Groups on Acidic Zeolite Catalysts. J. Am. Chem. Soc. 125, 15260-15267 (2003).

17. Dai, W. et al. Understanding the Early Stages of the Methanol-to-Olefin Conversion on HSAPO-34. ACS Catal. 5, 317-326 (2015).

18. Wang, C. et al. Experimental Evidence on the Formation of Ethene through Carbocations in Methanol Conversion over H-ZSM-5 Zeolite. Chem. - A Eur. J. 21, 12061-12068 (2015).

19. Chua, Y. T. \& Stair, P. C. An ultraviolet Raman spectroscopic study of coke formation in methanol to hydrocarbons conversion over zeolite H-MFI. J. Catal. 213, 39-46 (2003).

20. Beato, P., Schachtl, E., Barbera, K., Bonino, F. \& Bordiga, S. Operando Raman spectroscopy applying novel fluidized bed micro-reactor technology. Catal. Today 205, 128-133 (2013).

21. Signorile, M. et al. Topology-dependent hydrocarbon transformations in the methanol-tohydrocarbons reaction studied by operando UV-Raman spectroscopy. Phys. Chem. Chem. 
Phys. 20, 26580-26590 (2018).

22. Howe, R. F. et al. Reactions of Dimethylether in Single Crystals of the Silicoaluminophosphate STA-7 Studied via Operando Synchrotron Infrared Microspectroscopy. Top. Catal. 61, 199$212(2018)$.

23. Qian, Q. et al. Combined Operando UV/Vis/IR Spectroscopy Reveals the Role of Methoxy and Aromatic Species during the Methanol-to-Olefins Reaction over H-SAPO-34. ChemCatChem 6, 3396-3408 (2014).

24. Borodina, E. et al. Influence of the Reaction Temperature on the Nature of the Active and Deactivating Species during Methanol to Olefins Conversion over H-SSZ-13. ACS Catal. 5, 992-1003 (2015).

25. Goetze, J. et al. Insights into the Activity and Deactivation of the Methanol-to-Olefins Process over Different Small-Pore Zeolites As Studied with Operando UV-vis Spectroscopy. ACS Catal. 7, 4033-4046 (2017).

26. Lesthaeghe, D., Horré, A., Waroquier, M., Marin, G. B. \& Van Speybroeck, V. Theoretical Insights on Methylbenzene Side-Chain Growth in ZSM-5 Zeolites for Methanol-to-Olefin Conversion. Chem. - A Eur. J. 15, 10803-10808 (2009).

27. Forester, T. R. \& Howe, R. F. In situ FTIR studies of methanol and dimethyl ether in ZSM-5. J. Am. Chem. Soc. 109, 5076-5082 (1987).

28. Stair, P. C. The Application of UV Raman Spectroscopy for the Characterization of Catalysts and Catalytic Reactions. Adv. Catal. 51, 75-98 (2007).

29. Kim, H., Kosuda, K. M., Van Duyne, R. P. \& Stair, P. C. Resonance Raman and surface- and tip-enhanced Raman spectroscopy methods to study solid catalysts and heterogeneous catalytic reactions. Chem. Soc. Rev. 39, 4820-4844 (2010).

30. Stanley, A., Parker, A. W., Towrie, M. \& Matousek, P. Efficient Rejection of Fluorescence from Raman Spectra Using Picosecond Kerr Gating. Appl. Spectrosc. 53, 1485-1489 (1999).

31. Matousek, P., Towrie, M. \& Parker, A. W. Fluorescence background suppression in Raman spectroscopy using combined Kerr gated and shifted excitation Raman difference techniques. $J$. Raman Spectrosc. 33, 238-242 (2002).

32. Blaszkowski, S. R. \& van Santen, R. A. The Mechanism of Dimethyl Ether Formation from Methanol Catalyzed by Zeolitic Protons. J. Am. Chem. Soc. 118, 5152-5253 (1996).

33. Allotta, P. M. \& Stair, P. C. Time-Resolved Studies of Ethylene and Propylene Reactions in Zeolite H-MFI by In-Situ Fast IR Heating and UV Raman Spectroscopy. ACS Catal. 2, 24242432 (2012).

34. Socrates, G. Infrared and Raman Characteristic Group Frequencies. (John Wiley \& Sons, Ltd, 2001).

35. Ghysels, A. et al. Shape-Selective Diffusion of Olefins in 8-Ring Solid Acid Microporous Zeolites. J. Phys. Chem. C 119, 23721-23734 (2015).

36. Tinnemans, S. J., Kox, M. H. F., Nijhuis, T. A., Visser, T. \& Weckhuysen, B. M. Real time quantitative Raman spectroscopy of supported metal oxide catalysts without the need of an internal standard. Phys. Chem. Chem. Phys. 7, 211-216 (2005).

37. Bjørgen, M. et al. Conversion of methanol to hydrocarbons over zeolite H-ZSM-5: On the origin of the olefinic species. J. Catal. 249, 195-207 (2007).

38. Database of Zeolite Structures. Available at: http://www.iza-structure.org/databases/. 
39. Mores, D. et al. Space- and Time-Resolved In-situ Spectroscopy on the Coke Formation in Molecular Sieves: Methanol-to-Olefin Conversion over H-ZSM-5 and H-SAPO-34. Chem. - A Eur. J. 14, 11320-11327 (2008).

40. Borodina, E. et al. Influence of the Reaction Temperature on the Nature of the Active and Deactivating Species During Methanol-to-Olefins Conversion over H-SAPO-34. ACS Catal. 7, 5268-5281 (2017).

41. Hwang, A., Kumar, M., Rimer, J. D. \& Bhan, A. Implications of methanol disproportionation on catalyst lifetime for methanol-to-olefins conversion by HSSZ-13. J. Catal. 346, 154-160 (2017).

42. $\mathrm{Li}, \mathrm{Y} ., \mathrm{Yu}, \mathrm{J} ., \mathrm{Xu}, \mathrm{R}$. Criteria for zeolite frameworks realizable for target synthesis. Angew. Chem. 52, 1673-1677 (2013).

43. Li, Y. \& Yu, J. New stories of zeolite structures: Their descriptions, determinations, predictions, and evaluations. Chemical Reviews 114, 7268-7316 (2014).

\section{Acknowledgements}

This research has been performed with the use of facilities at the Research Complex at Harwell including Raman and TGA equipment. The authors would like to thank the Research Complex for access and support to these facilities and equipment. We would like to acknowledge the following EPSRC grants for funding: EP/K007467/1, EP/K014706/2, EP/K014668/1， EP/K014854/1， EP/K014714/1， EP/M013219/1， EP/S016481/1 and EP/S016481/1. STFC is acknowledged for the beam time on ULTRA for performing KerrGate experiments. Loredana Mantarosie (Johnson Matthey PLC) is also thanked for her assistance and expertise. Pavel Matousek and Tony Parker (STFC) are acknowledged for useful discussions. VVS, AH and MB, acknowledge the Research Foundation - Flanders (FWO), the Research Board of Ghent University (BOF), and funding from the European Union's Horizon 2020 research and innovation program (consolidator ERC Grant Agreement No. 647755 - DYNPOR (2015-2020)). The computational resources and services used were provided by Ghent University (Stevin Supercomputer Infrastructure) and the VSC (Flemish Supercomputer Center), funded by the Research Foundation - Flanders (FWO).

\section{Author Contributions}

I.L.G and A.M.B. conceived and coordinated the project, in close collaboration with V.V.S., M.T. and I.V.S. I.L.G. performed the operando Kerr-Gated Raman experiments with support from E.C., M.A.A., E.K.G., A.G. and I.V.S. The molecular simulations were performed by A.E.J.H., M.B., K.D.W. and V.V.S. I.L.G, E.C., A.E.J.H., V.V.S., K.D.W., A.M.B, M.T. and 
450 I.V.S. contributed to the data analysis and discussion. The manuscript was written by I.L.G., 451 E.C., A.E.J.H., V.V.S. and A.M.B, with input from all authors.

452

453 Competing Interests

454 The authors declare no competing interests.

455 
Methods

\section{Catalyst synthesis}

ZSM-5 zeolite $(\mathrm{Si} / \mathrm{Al}=15)$ was supplied by Zeolyst International in the ammonium form (CBV3024E), and the $\mathrm{H}$-form of the zeolite material was obtained by calcination in air at 550 ${ }^{\circ} \mathrm{C}\left(2{ }^{\circ} \mathrm{C} \cdot \mathrm{min}^{-1}\right.$ to $120{ }^{\circ} \mathrm{C}$, held for $30 \mathrm{~min}$; and $5^{\circ} \mathrm{C} \cdot \mathrm{min}^{-1}$ to $550{ }^{\circ} \mathrm{C}$, and $12 \mathrm{~h}$ at this temperature). SAPO-34 material is commercially available (ACS Material).

SSZ-13 zeolite $(\mathrm{Si} / \mathrm{Al}=15)$ was synthesized as described previously, ${ }^{44}$ but using static conditions. The sample was calcined in air (with the following temperature program: 1 ${ }^{\circ} \mathrm{C} \cdot \mathrm{min}^{-1}$ to $120^{\circ} \mathrm{C}$, held for $2.5 \mathrm{~h} ; 2.2{ }^{\circ} \mathrm{C} \cdot \mathrm{min}^{-1}$ to $350{ }^{\circ} \mathrm{C}$, and $3 \mathrm{~h}$ at this temperature; and finally $0.8{ }^{\circ} \mathrm{C} \cdot \mathrm{min}^{-1}$ to $580{ }^{\circ} \mathrm{C}$, and held for $3 \mathrm{~h}$ ). Identity and purity of the zeolite were verified by XRD. Further characterization of the material (i.e. XRD, chemical analysis, ${ }^{27} \mathrm{Al}$ NMR, $\mathrm{NH}_{3}-\mathrm{TPD}$, SEM and FT-IR) is reported in a previous study ${ }^{45}$ and not repeated herein.

\section{Operando Kerr-Gated Raman}

The Kerr-Gated Raman Spectrometer was used at the ULTRA Facility of the Central Laser Facility at the Rutherford Appleton Laboratory ${ }^{32-33}$. The laser source is a titanium sapphire laser which emits at $800 \mathrm{~nm}$. The beam is frequency doubled to $400 \mathrm{~nm}$ to produce the Raman probe beam. About $10 \mathrm{~mW}$ average power was delivered to samples in pulses of 3 ps at 10 $\mathrm{kHz}$ repetitions. The spot size of the beam at the sample is $100 \mu \mathrm{m}$ and the sample was rastered to minimize sample damage caused by the beam. $\mathrm{CS}_{2}$ was used as a Kerr medium, activated by the fundamental $800 \mathrm{~nm}$ beam of the laser. The arrival time of the excitation pulse at the sample is adjustable with respect to the gating pulse. Several time delays are measured at each acquisition to ensure that the optimal Raman signal is measured. Details on the ULTRA laser system and the Kerr-Gate setup are provided in the ESI.

The reaction was performed in a commercially available CCR1000 stage by Linkam Scientific. The cell consists of a ceramic cup, which holds the solid catalyst on top of a ceramic fabric filter. This is placed inside the cell with quartz windows, where an inner heating element controls the temperature of the catalyst. The outer surface of the cell is cooled by a water cooling system. Gases enter the cell flowing down the catalyst bed. Gases enter a chamber in the cell where they flow upwards and then down over the catalyst bed. The cell outlet is carried to a Pfeiffer OmniStar MS to analyze the reaction products.

$50 \mathrm{mg}$ of catalyst was used in the experiments. Prior to the reaction, the catalyst was activated at $550{ }^{\circ} \mathrm{C}\left(5{ }^{\circ} \mathrm{C} \mathrm{min}^{-1}\right)$ under a $20 \% \mathrm{O}_{2} / \mathrm{He}$ flow for 1 hour. He was flushed through the 
system to remove all $\mathrm{O}_{2}$, and the catalyst cooled to $100{ }^{\circ} \mathrm{C}$. He was used as a carrier gas at a flow of $30 \mathrm{ml} \mathrm{min}^{-1}$. Methanol was injected into the system by use of a HPLC pump set to 1.7 $\mu 1 \mathrm{~min}^{-1}$ corresponding to a WHSV of $1.6 \mathrm{~h}^{-1}$, and flows allowed to equilibrate by monitoring the signals from the MS. Subsequently, the catalyst was heated up to $450{ }^{\circ} \mathrm{C}$ with a rate of 1 ${ }^{\circ} \mathrm{C} \min ^{-1}$. After the experiment, the zeolite catalyst was cooled rapidly to $30{ }^{\circ} \mathrm{C}$ by the cooling system of the cell, to quench the reaction.

Prior to beginning the reaction, Raman spectra of the calcined catalyst samples were measured, using 5 accumulations of 20 seconds exposure to give a total $100 \mathrm{~s}$ exposure time. During the reaction, 1 accumulation of 20 seconds exposure was used per measurement. This allows time resolution of 20 seconds, to allow real-time investigation of the HP intermediates. During temperature ramp experiments, measurements were taken every $10{ }^{\circ} \mathrm{C}$.

\section{Theoretical calculations}

MD simulations were carried out with the CP2K simulation package (version 3.0$)^{46}$ at a Density Functional Theory (DFT) level of theory combined with a Gaussian and plane wave basis set (GPW). ${ }^{47}$ The revPBE-D3 functional ${ }^{48}$ with a DZVP-GTH basis set and pseudopotentials were selected, ${ }^{49}$ as done previously, ${ }^{50}$ with a plane wave cutoff of $400 \mathrm{Ry}$. All simulations were performed in a periodic zeolite unit cell representing the CHA topology containing $36 \mathrm{~T}$-sites and two Brønsted acid sites, corresponding to a $\mathrm{Si} / \mathrm{Al}$ ratio of 17 -which is close to the experimental value of 15 .

A large set of MD simulations were performed encompassing the empty zeolite, a methanol loaded zeolite and a broad variety of alkene, polyene and aromatic adsorbates (Fig. S5). For each guest molecule, cell parameters were obtained by computing time-averaged values from a $50 \mathrm{ps}$ NPT MD simulation at $350{ }^{\circ} \mathrm{C}$ and atmospheric pressure. The temperature and pressure were controlled via a chain of 5 Nosé-Hoover thermostats and an MTK barostat, respectively. ${ }^{51}$ An integration time step of 0.5 fs was applied. Production MD runs used as input for the Raman spectra and mobility analysis were carried out in the NVT ensemble at $350{ }^{\circ} \mathrm{C}$ for $40 \mathrm{ps}$, with the unit cell parameters as listed in Table S2, preceded by an equilibration of $2.5 \mathrm{ps}$.

Theoretical Raman spectra were obtained via Fourier transforms of the autocorrelation functions of the polarizability tensor. ${ }^{52}$ Therefore, the polarizability tensor was derived every 2 fs through a finite difference approach. ${ }^{53}$ For more details we refer to the ESI. 


\section{Data Availability}

Source data for Figs. 1-6, S3-S4, S38-42 are provided with the paper. Example CP2K input files and processing scripts are available from the public GitHub online repository at https://github.com/AlexanderHoffman/supporting-info. Due to the size of the MD trajectories and $\mathrm{CP} 2 \mathrm{~K}$ output files, they are available upon author request.

\section{Moliner, M., Franch, C., Palomares, E., Grill, M. \& Corma, A. Cu-SSZ-39, an active and hydrothermally stable catalyst for the selective catalytic reduction of NOx. Chem. Commun. 48, 8264-8266 (2012).}

45. Lezcano-Gonzalez, I. et al. Determining the storage, availability and reactivity of $\mathrm{NH}_{3}$ within Cu-Chabazite-based Ammonia Selective Catalytic Reduction systems. Phys. Chem. Chem. Phys. 16, 1639-1650 (2014).

46. Hutter, J., Iannuzzi, M., Schiffmann, F. \& VandeVondele, J. cp2k: atomistic simulations of condensed matter systems. Wiley Interdiscip. Rev. Comput. Mol. Sci. 4, 15-25 (2014).

47. Lippert, G., Hutter, J. \& Parrinello, M. The Gaussian and augmented-plane-wave density functional method for ab initio molecular dynamics simulations. Theor. Chem. Accounts Theory, Comput. Model. (Theoretica Chim. Acta) 103, 124-140 (1999).

48. Yang, K., Zheng, J., Zhao, Y. \& Truhlar, D. G. Tests of the RPBE, revPBE, $\tau$-HCTHhyb, $\omega \mathrm{B} 97 \mathrm{X}-\mathrm{D}$, and MOHLYP density functional approximations and 29 others against representative databases for diverse bond energies and barrier heights in catalysis. J. Chem. Phys. 132, 164117 (2010).

49. Goedecker, S., Teter, M. \& Hutter, J. Separable dual-space Gaussian pseudopotentials. Phys. Rev. B 54, 1703-1710 (1996).

50. Cnudde, P. et al. How Chain Length and Branching Influence the Alkene Cracking Reactivity on H-ZSM-5. ACS Catal. 8, 9579-9595 (2018).

51. Frenkel, D. \& Smit, B. Understanding Molecular Simulation. (Academic Press: San Diego, 2002).

52. Luber, S., Iannuzzi, M. \& Hutter, J. Raman spectra from $a b$ initio molecular dynamics and its application to liquid $S$-methyloxirane. J. Chem. Phys. 141, 094503 (2014).

53. Thomas, M., Brehm, M., Fligg, R., Vöhringer, P. \& Kirchner, B. Computing vibrational spectra from ab initio molecular dynamics. Phys. Chem. Chem. Phys. 15, 6608-6622 (2013). 